\title{
Diagnostic Evaluation of Foreign Body Aspiration in Children
}

\author{
(1) Ali Onur Erdem, (1) Barlas Etensel, (1) Mesut Yazıcı, @ Sezen Karaca Özkısacık \\ Adnan Menderes University Faculty of Medicine, Department of Pediatric Surgery, Aydın, Turkey
}

\begin{abstract}
Aim: The aim of this study was to evaluate the place of clinical symptoms and findings, and radiological imaging in the diagnosis of foreign body aspiration (FBA) in children, and to investigate the validity of existing diagnostic algorithms.

Materials and Methods: The medical records of 120 children with a diagnosis of FBA who underwent rigid bronchoscopy were examined. The sensitivity and specificity of the parameters used in the diagnosis of FBA and their predictive values were calculated.

Results: $78.9 \%$ of the patients were younger than 3 years and $63.8 \%$ were male. The peak incidence was 18 months. The rate of negative bronchoscopy was $30.8 \%$ and $87.3 \%$ of these patients were younger than 3 years. It was observed that the value with the highest diagnostical sensitivity and diagnostic accuracy (DA) rate (sensitivity; $92.54 \%$ and DA; $85.7 \%$ ) had positivity for any of the radiological findings, and among these, there was the detection of one-sided excessive ventilation on chest X-ray (sensitivity; $70.0 \%$ and DA; $70.01 \%$ ). Patients with positivity of any of the radiological findings who additionally had wheezing together with cyanosis or dyspnea had $100 \%$ specificity.

Conclusion: Particularly male children under the age of 3 years have an increased risk of FBA. Neither clinical symptoms nor radiological findings alone are sufficiently specific and sensitive in the diagnosis of FBA. The most important factor in the decision to perform bronchoscopy is the evaluation of radiological findings together with physical examination and history.
\end{abstract}

Keywords: Foreign body aspiration, children, algorithms

\section{Introduction}

Foreign body aspiration (FBA) is the most common cause of death due to home accidents in children under 6 years of age, and is one of the major causes of mortality and morbidity in childhood (1-3). The clinical features of FBA can be life-threatening airway obstruction or lighter manifestations such as recurrent pulmonary infection, wheezing, and cough (4). Therefore, it is important to diagnose immediately and provide appropriate treatment in order to reduce the mortality and morbidity rates. Foreign bodies can be removed by rigid or flexible bronchoscopy, but the prerequisite for this is the suspicion of foreign body presence (5).

In this study, we aimed to evaluate the place of clinical symptoms, findings and radiological imaging in the diagnosis of FBA in children, and to investigate the validity of existing diagnostic algorithms.

\section{Materials and Methods}

Before starting the study, (approval no: 2018/1416) was obtained from the non-interventional clinical research ethics committee of Adnan Menderes University. In this

\section{Address for Correspondence}

Asst. Prof. Ali Onur Erdem, Adnan Menderes University Faculty of Medicine Department of Pediatric Surgery, Aydın, Turkey Phone: +905053882174 E-mail: aoerdem@adu.edu.tr ORCID: orcid.org/0000-0002-9584-4200

Received: 29.10.2019 Accepted: 12.02 .2020

${ }^{(0)}$ Copyright 2021 by Ege University Faculty of Medicine, Department of Pediatrics and Ege Children's Foundation The Journal of Pediatric Research, published by Galenos Publishing House. 
study, 120 patients who underwent rigid bronchoscopy for suspected FBA in Adnan Menderes University Faculty of Medicine, Department of Pediatric Surgery between 2000-2019, were evaluated retrospectively. All patients were evaluated in terms of age, gender, time of admission, cough or symptoms of cyanosis (choking) in the patient history, wheezing in physical examination, some symptoms of dyspnea; nasal flaring, the presence of intercostal and suprasternal retractions, and the presence of excessive ventilation and pneumonic infiltration on chest $X$-ray. The relationship between all findings and foreign body detection in bronchoscopy was evaluated statistically.

\section{Statistical Analysis}

Statistical analysis were carried out with SPSS v12.0 statistical software (SPSS, Chicago, IL). The specific parameters of diagnostic accuracy (DA), specificity and sensitivity were analyzed statistically. $\mathrm{P}<0.05$ was considered significant.

\section{Results}

A total of 120 patients underwent rigid bronchoscopy for suspected FBA between January 2000 and March 2019 at Adnan Menderes University Faculty of Medicine, Department of Pediatric Surgery. Foreign bodies in the tracheobronchial tree were detected in $71(59.2 \%)$, and were not detected in $49(40.8 \%)$ patients. The mean age of all patients at admission was 2 years ( 5 months- 14 years of age).

Fifty-four (76.0\%) patients with foreign bodies and 40 (90.9\%) patients without foreign bodies were under 3 years of age. Seventy-six (63.3\%) patients who underwent bronchoscopy were male and 44 patients $(36.6 \%)$ were female. Twenty-six (36.6\%) patients with foreign bodies were female and $45(63.4 \%)$ were male; 19 (38.8\%) patients without foreign bodies were female and 30 (61.2\%) were male. There was no significant difference between gender distribution and the presence of foreign bodies in the tracheobronchial tree ( $p>0.05)$. The mean admission time was 4 days (minimum; 1 day - maximum; 180 days).

Forty-five (63.4\%) patients with foreign bodies detected via bronchoscopy had cough, 23 (32.4\%) had cyanosis (choking), and $18(25 \%)$ had both cough and cyanosis (choking).

A positive history of cough in 21 (46.7\%), cyanosis (choking) in 18 (40.9\%), cough and cyanosis (choking) in 16 (36.4\%) patients without foreign bodies was observed.

The presence of cough in history sensitivity was $64.29 \%$, whereas the presence of cyanosis (choking) sensitivity was $32.86 \%$. When positive predictive values $\left(\mathrm{PV}^{+}\right)$were examined, it was found that both values were high, although the cough value $(68.18 \%)\left(\mathrm{PV}^{+}\right)$was higher than the cyanosis value $(56.10 \%)$. There were no significant relations between the presence of foreign bodies and cough or cyanosis (choking) in the patient history ( $p>0.05$ ).

Thirty-eight $(53.5 \%)$ patients with foreign bodies detected in bronchoscopy had dyspnea, 21 (29.6\%) had wheezing, and 18 (25\%) had dyspnea and wheezing.

Eighteen (40.9\%) patients without foreign bodies had dyspnea, 6 (13.6\%) had wheezing, and 4 (9.1\%) had dyspnea and wheezing. While the sensitivity and specificity of dyspnea were $54.29 \%$ and $63.27 \%$ respectively, the sensitivity and specificity of wheezing were $30.0 \%$ and $87.76 \%$ respectively. When evaluated statistically, there was no significant relationship between wheezing and the detection of foreign bodies ( $p>0.05$ ), but there was a significant relationship between dyspnea and the detection of foreign bodies $(p<0.05)$.

When the preoperative PA chest X-ray was evaluated, it was observed that $5(7.01 \%)$ of the patients with foreign body had normal radiological findings, 24 (33.80\%) had pneumonic infiltration, and 49 (69.01\%) had excessive ventilation.

In the preoperative radiological evaluation of the patients without foreign bodies, it was observed that $9(20.5 \%)$ patients had normal findings, 12 (27.3\%) had excessive ventilation, and 9 (20.45\%) had infiltration on PA chest X-ray. Foreign bodies were detected in 24 (33.80\%) patients with infiltration on preoperative chest $X$-ray, and when evaluated statistically, there was a significant relationship between the detection of foreign bodies in the tracheobronchial tree and the presence of infiltration on chest X-ray $(p<0.05)$. However, only $9(20.45 \%)$ patients without foreign bodies had infiltration on preoperative chest X-ray. In other words, on the preoperative chest X-ray of those patients who underwent bronchoscopy and had no foreign bodies, the rate of absence of infiltration was high (90.9\%).

When the preoperative chest X-ray was evaluated in terms of excessive ventilation, 49 (69.0\%) patients with foreign bodies and 12 (27.01\%) patients without foreign bodies had excessive infiltration and a statistically significant relationship was found $(p<0.05)$ (Figure 1).

Statistical analyses are shown in Table I. Cough, cyanosis, wheezing and dyspnea were used as diagnostic parameters. Pneumonic infiltration, excessive ventilation, and the presence of any radiological findings were used as 
radiological tests. It was observed that the value with the highest diagnostical sensitivity and DA rate (sensitivity; 92.54\% and DA; $85.7 \%$ ) had positivity of any of the radiological findings, and among these, there was detection of one-sided excessive ventilation on the chest $\mathrm{X}$-ray

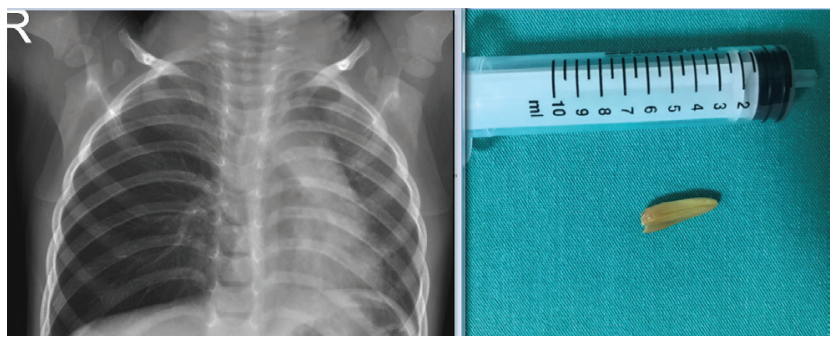

Figure 1. Chest $\mathrm{X}$-ray with non-radiopaque foreign bodies and removed object (sensitivity; 70.0\% and DA; 70.01\%). When the specificities of these findings were examined, it was observed that the specificity of wheezing was $87.76 \%$, and the highest specificity values other than wheezing were found to be infiltration on the chest X-ray at $81.63 \%$ and the detection of one-sided excessive ventilation at $80.33 \%$.

Table II shows coexisting rates of two or three of the parameters evaluated in the patient history, physical examination and radiological findings, and their statistical values. In cases of cough with dyspnea or cough with wheezing, the specificity values were $81.25 \%$ and $92.31 \%$, respectively. Likewise, sensitivity values were found to be high in cases of presence of cough with excessive ventilation, which is one of the radiological findings, and these values were $83.33 \%$. Among these, the highest DA was found to be the presence of dyspnea with cough

Table I. Statistical values of history, physical examination and radiological findings individually

\begin{tabular}{|c|c|c|c|c|c|c|c|c|c|c|c|c|c|c|}
\hline & \multicolumn{2}{|c|}{ Cough } & \multicolumn{2}{|c|}{ Cyanosis } & \multicolumn{2}{|c|}{ Wheezing } & \multicolumn{2}{|c|}{ *Dyspnea } & \multicolumn{2}{|c|}{ *Infiltration } & \multicolumn{2}{|l|}{${ }^{*} \mathrm{EV}$} & \multicolumn{2}{|c|}{ Radiology } \\
\hline & + & - & + & - & + & - & + & - & + & - & + & - & + & - \\
\hline FBA+ & 45 & 25 & 23 & 47 & 21 & 49 & 38 & 32 & 24 & 46 & 49 & 21 & 62 & 5 \\
\hline FBA- & 21 & 28 & 18 & 31 & 6 & 43 & 18 & 31 & 9 & 40 & 12 & 37 & 40 & 9 \\
\hline DA\% & \multicolumn{2}{|l|}{55.5} & \multicolumn{2}{|c|}{35.4} & \multicolumn{2}{|l|}{22.7} & \multicolumn{2}{|l|}{47.1} & \multicolumn{2}{|l|}{34.3} & \multicolumn{2}{|c|}{70.0} & \multicolumn{2}{|l|}{85.7} \\
\hline Sensitivity \% & \multicolumn{2}{|c|}{64.29} & \multicolumn{2}{|c|}{32.86} & \multicolumn{2}{|c|}{30.00} & \multicolumn{2}{|c|}{54.29} & \multicolumn{2}{|c|}{34.29} & \multicolumn{2}{|c|}{70.00} & \multicolumn{2}{|l|}{92.54} \\
\hline Specificity \% & \multicolumn{2}{|l|}{57.14} & \multicolumn{2}{|c|}{63.27} & \multicolumn{2}{|c|}{87.76} & \multicolumn{2}{|c|}{63.27} & \multicolumn{2}{|c|}{81.63} & \multicolumn{2}{|c|}{75.51} & \multicolumn{2}{|l|}{18.37} \\
\hline $\mathrm{PV}^{+}$ & \multicolumn{2}{|c|}{68.18} & \multicolumn{2}{|c|}{56.10} & \multicolumn{2}{|c|}{77.78} & \multicolumn{2}{|c|}{67.86} & \multicolumn{2}{|c|}{72.73} & \multicolumn{2}{|c|}{80.33} & \multicolumn{2}{|l|}{60.78} \\
\hline PV & \multicolumn{2}{|c|}{52.83} & \multicolumn{2}{|c|}{39.74} & 46.7 & & 49.2 & & 46.5 & & 63.7 & & 64.29 & \\
\hline
\end{tabular}

Table II. Coexisting rates of two or three of the parameters evaluated in history, physical examination and radiological findings, and their statistical values

\begin{tabular}{|c|c|c|c|c|c|c|c|c|c|c|c|c|c|c|c|c|c|c|c|c|c|c|c|c|}
\hline & \multicolumn{2}{|l|}{$\mathrm{Cl}$} & \multicolumn{2}{|c|}{ CEV } & \multicolumn{2}{|c|}{ Cyl } & \multicolumn{2}{|c|}{ CyEV } & \multicolumn{2}{|c|}{ WI } & \multicolumn{2}{|c|}{ WEV } & \multicolumn{2}{|l|}{ DI } & \multicolumn{2}{|c|}{ DEV } & \multicolumn{2}{|c|}{ CW } & \multicolumn{2}{|l|}{$C D$} & \multicolumn{2}{|c|}{ CyW } & \multicolumn{2}{|c|}{ CyD } \\
\hline & + & - & + & - & + & - & + & - & + & - & + & - & + & - & + & - & + & - & + & - & + & - & + & - \\
\hline FBA+ & 17 & 18 & 30 & 6 & 9 & 32 & 16 & 14 & 9 & 34 & 16 & 16 & 12 & 20 & 29 & 12 & 18 & 22 & 24 & 11 & 5 & 31 & 18 & 27 \\
\hline FBA- & 2 & 21 & 6 & 22 & 2 & 24 & 4 & 23 & 2 & 36 & 4 & 35 & 3 & 25 & 3 & 22 & 2 & 24 & 3 & 13 & 1 & 26 & 9 & 22 \\
\hline DA \% & \multicolumn{2}{|c|}{$\begin{array}{l}60 \\
(46-72)\end{array}$} & \multicolumn{2}{|c|}{$\begin{array}{l}56 \\
(43-68)\end{array}$} & \multicolumn{2}{|c|}{$\begin{array}{l}61 \\
(48-72)\end{array}$} & \multicolumn{2}{|c|}{$\begin{array}{l}52 \\
(34-71)\end{array}$} & \multicolumn{2}{|c|}{$\begin{array}{l}53 \\
(41-64)\end{array}$} & \multicolumn{2}{|c|}{$\begin{array}{l}45 \\
(33-57)\end{array}$} & \multicolumn{2}{|c|}{$\begin{array}{l}53 \\
(40-66)\end{array}$} & \multicolumn{2}{|c|}{$\begin{array}{l}62 \\
(49-73)\end{array}$} & \multicolumn{2}{|c|}{$\begin{array}{l}60 \\
(47-72)\end{array}$} & \multicolumn{2}{|c|}{$\begin{array}{l}68 \\
(54-80)\end{array}$} & \multicolumn{2}{|c|}{$\begin{array}{l}57 \\
(44-69)\end{array}$} & \multicolumn{2}{|c|}{$\begin{array}{l}59 \\
(47-70)\end{array}$} \\
\hline $\begin{array}{l}\text { Sensitivity } \\
\%\end{array}$ & \multicolumn{2}{|c|}{48.57} & \multicolumn{2}{|c|}{83.33} & \multicolumn{2}{|c|}{21.95} & \multicolumn{2}{|c|}{53.33} & \multicolumn{2}{|c|}{20.93} & \multicolumn{2}{|c|}{50.00} & \multicolumn{2}{|c|}{37.50} & \multicolumn{2}{|c|}{70.73} & 45.0 & & 68.5 & & 13. & & 40.0 & \\
\hline $\begin{array}{l}\text { Specificity } \\
\%\end{array}$ & 91.3 & & 78.5 & & 92. & & 85.1 & & 94.7 & & 89.74 & & 89.2 & & 88.0 & & 92.3 & & 81.25 & & & & 70.9 & \\
\hline $\mathrm{PV}^{+} \%$ & 89.4 & & 83.3 & & $81 . \varepsilon$ & & 80.0 & & 81.8 & & 80.0 & & 80.0 & & 90.6 & & 90.0 & & 88.8 & & & & 66.6 & \\
\hline PV-\% & 53.8 & & 78.5 & & 42. & & 62.1 & & 51.4 & & 68.63 & & 55.5 & & 64.7 & & 52.17 & & 54.17 & & & & 44.9 & \\
\hline
\end{tabular}


(68\%), followed by the presence of infiltration with cough (60\%). Specificity of infiltration together with the presence of cough, cyanosis, wheezing or dyspnea was found to be $90 \%$ or more. The presence of wheezing with cyanosis specificity was calculated to be $96.30 \%$. When patients with radiologically pneumonic infiltration or excessive ventilation were evaluated with these data, if the radiological findings of patients with cyanosis and wheezing and patients with wheezing and dyspnea were positive, their specificity were 100\% (Table III).

Patients with cyanosis (choking) and/or cough history, wheezing and/or dyspnea on physical examination, were bronchoscopically evaluated for the presence of foreign bodies, even if they had suspicious history and excessive ventilation on chest $X$-ray. A total of 120 patients underwent bronchoscopy and foreign bodies were detected in 71 . Foreign bodies were detected in the right main bronchus in $34(47.88 \%)$ patients with foreign bodies, in the trachea in $13(18.30 \%)$ and in the left main bronchus in 24 (33.80\%) (Table IV). When evaluated statistically, detecting foreign bodies in the right main bronchus was significantly high $(p<0.05)$. In 49 patients, no foreign bodies were detected in bronchoscopy, but 12 of these 49 patients had mucus plugs in the bronchi, and washing with saline was performed.
The other 37 patients (30.8\%) were considered as negative bronchoscopy. In patients with mucus plugs, clinical and radiological findings significantly improved after bronchoscopic washing and they were discharged without any further problems.

\section{Discussion}

Foreign bodies were detected in 71 (59.2\%) patients, and no foreign bodies were detected in $49(40.8 \%)$ patients. The mean age was 2 years ( 7 months - 14 years), the majority $(n=94,78.3 \%)$ were under the age of 3 years and it was found that more males had foreign bodies. All these data were consistent with the literature $(1,6,7)$. According to all these data, we can say that children under 3 years of age have the highest risk for FBA.

As the distal airways are narrower, aspirated foreign bodies are most commonly located in the proximal airways and are directed to the right main bronchus as it has more vertical course and larger diameter than the left (5,7-9). In our patients, the most common site was the right main bronchus (47.88\%), the second most common was the left main bronchus (33.80\%) and the third was the trachea (18.30\%). It was observed that the right main bronchus rate was significantly higher $(p<0.05)$.

Table III. Coexisting rates of two or three of the parameters evaluated in history, physical examination and radiological findings, and their statistical values

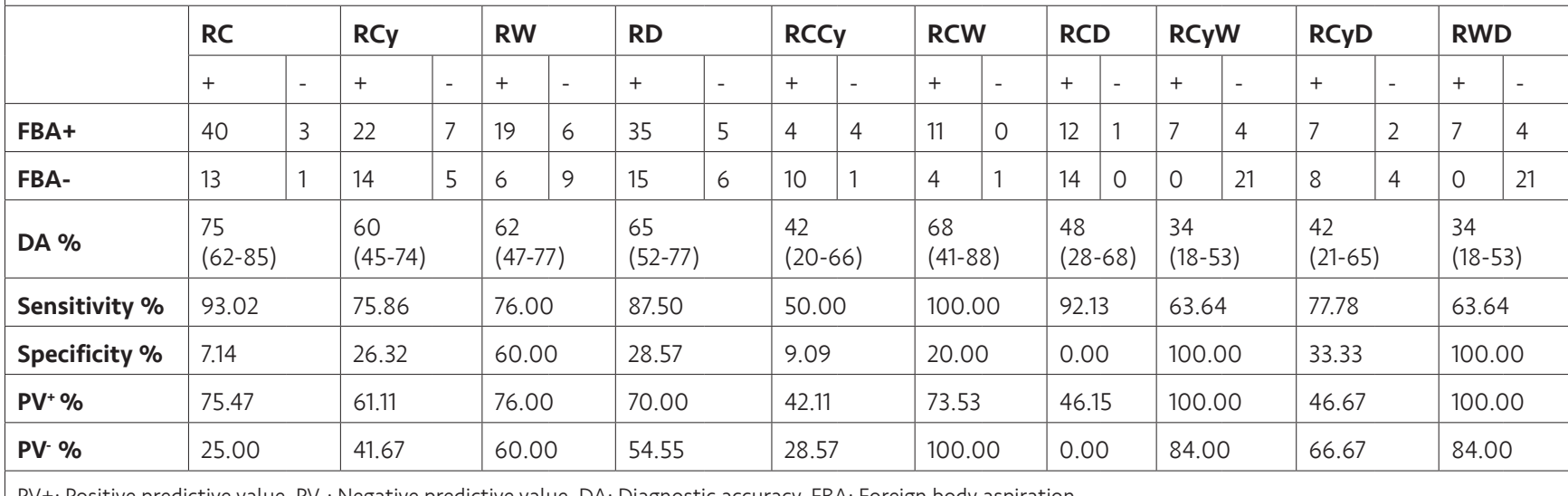

PV+: Positive predictive value, PV-: Negative predictive value, DA: Diagnostic accuracy, FBA: Foreign body aspiration

Table IV. Patients whose foreign bodies were removed by bronchoscopy and the locations of foreign bodies

\begin{tabular}{|l|l|l|}
\hline Location of foreign body & Number of patients & $\%$ \\
\hline *Right main bronchus & 34 & 47.88 \\
\hline Trachea & 13 & 18.30 \\
\hline Left main bronchus & 24 & 33.80 \\
\hline Total & 71 & 100 \\
\hline${ }^{*}<0.05$ & & \\
\hline
\end{tabular}


In a review of Metrangelo et al. (7), in which they presented their 8-year experience in FBA in children, they reported that the foreign bodies were most frequently encountered in the right main bronchus, but found that the encountering rate in the left main bronchus was very close to the rate of the right. They explained this as bronchial anatomy alone was not effective on the location of foreign bodies, these foreign bodies can be displaced by cough or ventilatory effort. In our patients, the foreign bodies encountering rate in the left main bronchus was second, and this can be explained by the fact that foreign bodies can be displaced by cough or ventilatory efforts, and this in line with the results of Metrangelo et al. (7).

Black et al. (4) retrospectively evaluated 548 patients who underwent bronchoscopy with suspected FBA and reported that choking, cough and wheezing were present in $95 \%$ of these patients, and bronchoscopy was required. In another study, it was reported that especially in patients with choking history, this was the most valuable data for diagnosis, and cough and wheezing were present in $95 \%$ of the patients (7). When we evaluated our patients in terms of cough, cyanosis (choking), wheezing and dyspnea presence; cough was the parameter with the highest sensitivity and DA (64.29\%), followed by dyspnea at a sensitivity rate of $54.29 \%$. When examined for their specificities, the highest value was found to be wheezing at $87.76 \%$, followed by cyanosis (choking) and dyspnea at $63.27 \%$. However, in the statistical evaluation of all these data, only dyspnea was found to be significantly high in patients with foreign bodies $(p<0.05)$. We also attributed the fact that such a small number of patients, in discordance with literature, had cyanosis (choking) history because aspiration occurred at a time when the family was not with the child or the family's attention was distracted. These results suggest that the presence of dyspnea among the clinical findings supports suspected FBA.

In $49(40.8 \%)$ patients who underwent rigid bronchoscopy due to FBA, no foreign bodies were found. According to the literature, the detection range of foreign bodies in children with suspected FBA is very wide (25\%$90 \%$ ). In Even et al.'s (10) study, foreign bodies were found in $57 \%$ of 98 children, and it was indicated that this ratio, which is close to the ratio in our study, is in accordance with the literature (11). Similarly, Oncel et al. (12) reported in their study that no foreign bodies were found during rigid bronchoscopy at the rate of $25.7 \%$. We think that the negative bronchoscopy rate is similar due to the vital risks that may occur usually in emergency conditions if bronchoscopy, a procedure that must be decided upon quickly, is not performed. It has been reported in the literature that computed tomography can also be a good alternative in the suspicion of foreign body diagnosis, and even can be used with a DA rate of $94.5 \%$. Therefore, in our retrospective series, the FBA detection rate in bronchoscopy is in accordance with the literature. It can be said that the negative bronchoscopy rate may be increased due to vital risks that occur in emergency conditions if bronchoscopy, a procedure that must be decided upon quickly, is not performed. It has been stated in the literature that computed tomography can be a good alternative and even be used at a DA rate of $94.5 \%$ (13). No computed tomography was used diagnostically in our patient series. Considering radiation exposure in the pediatric age group, we think that using computed tomography routinely in the diagnosis of FBA is arguable.

However, considering the risks of anesthesia and the procedure during bronchoscopy, the place of computed tomography in the diagnostic algorithm should be reassessed with prospective studies.

If cyanosis (choking) history is positive, bronchoscopy is recommended in the diagnostic approach to patients with suspected FBA, even if physical examination and radiographic findings are negative $(2,14,15)$. When we evaluated our patients in the light of this information, there were two patients who underwent bronchoscopy who had negative physical examination and radiological findings, and positive cyanosis (choking) history, and they had no foreign bodies. Again, it was observed that the sensitivity value of history positivity, which is the most valuable diagnostic criterion when compared to the literature information, was $25 \%$ lower in our cases. In parallel with this information, in discordance with the literature, we thought that positive history alone would not be sufficient, and we evaluated the relationship between history together with any physical examination or radiological findings and the presence of a foreign bodies. In our patients, if there was wheezing as a physical examination finding with cyanosis (choking) history, or a history of wheezing and positivity of any radiological findings, the specificity was found to be $100 \%$. In this case, according to our data, we think that history alone may not be sufficient for the indication of bronchoscopy, and bronchoscopy should be performed if any radiological findings (pneumonic infiltration or excessive ventilation) are positive.

Metallic foreign bodies can be diagnosed directly by $X$-ray. However, since foreign bodies are non-opaque, the diagnosis is based on secondary findings (16) (Figure 2). 

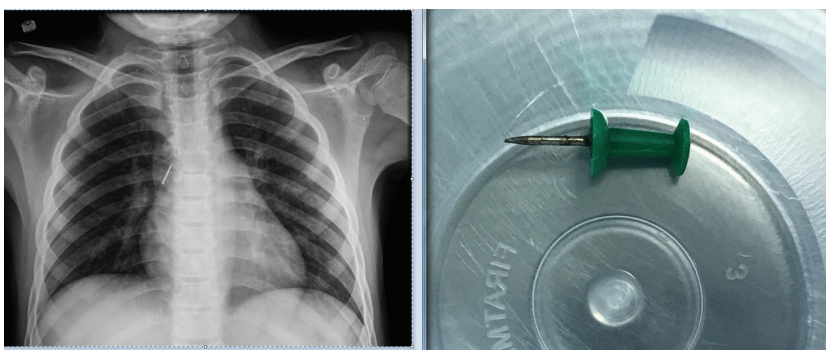

Figure 2. Chest $\mathrm{X}$-ray with radiopaque foreign bodies and removed object

In addition, the DA rate (85.70\%) was found to be high in patients with any radiological findings detected in chest X-ray. The detection sensitivity (92.50\%) of the radiological findings was also found to be high. However, no foreign bodies were observed in 40 (39\%) patients with pathological findings. With these findings, radiological finding detection alone is not sufficient to indicate bronchoscopy. However, it is seen that there is a $100 \%$ specificity value with history and physical examination findings.

\section{Conclusion}

If those patients with suspicious history, which is the most important parameter for diagnosis in FBAs, also have dyspnea and pneumonic infiltration on chest X-ray, the possibility of FBA presence should definitely be considered, even if the first thing which comes to mind in diagnosis is pneumonia. We believe that these patients with suspicious history should be evaluated bronchoscopically if they have a suspicious history together with wheezing and/ or pneumonic infiltration on chest X-ray and/or excessive ventilation.

\section{Ethics}

Ethics Committee Approval: Ethics approval was obtained from the Non-interventional Clinical Research Ethics Committee of Adnan Menderes University (approval no: 2018/1416).

Informed Consent: Retrospective study.

Peer-review: Externally peer-reviewed.

\section{Authorship Contributions}

Surgical and Medical Practices: A.O.E., B.E., M.Y., S.K.Ö., Concept: A.O.E., B.E., M.Y., S.K.Ö., Design: A.O.E., B.E., M.Y., S.K.Ö., Data Collection or Processing: A.O.E., B.E., S.K.Ö., Analysis or Interpretation: A.O.E., B.E., M.Y., S.K.Ö., Literature Search: A.O.E., B.E., M.Y., S.K.Ö., Writing: A.O.E., B.E., M.Y., S.K.Ö.
Conflict of Interest: None of the authors had conflict of interest.

Financial Disclosure: The authors declared that this study received no financial support.

\section{References}

1. Brkic F, Umihanic S. Tracheobronchial foreign bodies in children. Experience at ORL clinic Tuzla, 1954-2004. Int J Pediatr Otorhinolaryngol 2007; 71:909-15.

2. Paksu S, Paksu MS, Kilic M, et al. Foreign body aspiration in childhood: evaluation of diagnostic parameters. Pediatr Emerg Care 2012; 28:259-64.

3. Skoulakis CE, Doxas PG, Papadakis CE, et al. Bronchoscopy for foreign body removal in children. A review and analysis of 210 cases. Int J Pediatr Otorhinolaryngol 2000; 53:143-8.

4. Black RE, Johnson DG, Matlak ME. Bronchoscopic removal of aspirated foreign bodies in children. J Pediatr Surg 1994; 29:6824.

5. Latifi X, Mustafa A, Hysenaj Q. Rigid tracheobronchoscopy in the management of airway foreign bodies: 10 years experience in Kosovo. Int J Pediatr Otorhinolaryngol 2006; 70:2055-9.

6. Ciftci AO, Bingol-Kologlu $M$, Senocak ME, Tanyel FC, Büyükpamukçu N. Bronchoscopy for evaluation of foreign body aspiration in children. J Pediatr Surg 2003; 38:1170-6.

7. Metrangelo S, Monetti C, Meneghini L, Zadra N, Giusti F. Eight years' experience with foreign-body aspiration in children: what is really important for a timely diagnosis? ) Pediatr Surg 1999; 34:1229-31.

8. Chiu CY, Wong KS, Lai SH, Wu CT. Factors predicting early diagnosis of foreign body aspiration in children. Pediatr Emerg Care 2005; 21:161-4

9. Etensel B, Erdem AO, Özkisacık S, Çoşkun Ö, Gürsoy MH. Current approaches to foreign body aspiration in children in the light of 8 years of clinical experience. Van Med I 2015; 22:90-5.

10. Even L, Heno N, Talmon Y, Zonis Z, Kugelman A. Diagnostic evaluation of foreign body aspiration in children: a prospective study. J Pediatr Surg 2005; 40:1122-7.

11. Schmidt H, Manegold BC. Foreign body aspiration in children. Surg Endosc 2000; 14:644-8.

12. Oncel M, Sunam GS, Ceran S. Tracheobronchial aspiration of foreign bodies and rigid bronchoscopy in children. Pediatr Int 2012; 54:532-5.

13. Gang W, Zhengxia P, Hongbo L, et al. Diagnosis and treatment of tracheobronchial foreign bodies in 1024 children. I Pediatr Surg 2012; 47:2004-10.

14. Adjeso T, Damah MC, Murphy IP, Anyomih TTK. foreign body aspiration in northern ghana: a review of pediatric patients. Int J Otolaryngol 2017; 2017:1478795.

15. Bolliger CT Mathur PN. .Prog Respir Res. Basel, Karger. Interventional Bronchoscopy. Cape Town; 2000:96-107.

16. Lukse R, Winter RC, Pollock AN. Metallic foreign body aspiration: case of a blowgun dart in the left main stem bronchus. Pediatr Emerg Care 2014; 30:586-7. 